\section{Treatment patterns of ranibizumab intravitreal injection and dexamethasone intravitreal implant for retinal vein occlusion in the USA}

S Nghiem-Buffet' ${ }^{1}$ S Baillif², S Regnier ${ }^{3}$, A Skelly4, $\mathrm{N} \mathrm{Yu^{5 } \text { and A Sodi }}{ }^{6}$

\begin{abstract}
Purpose Ranibizumab, an anti-vascular endothelial growth factor, and dexamethasone, a corticosteroid, have been shown to be effective in treating macular oedema secondary to retinal vein occlusion (RVO) (central RVO (CRVO) and branch RVO (BRVO)). Their real-world usage, however, has yet to be compared. We therefore evaluated ophthalmology visits for both drugs using US patient-level data. Methods The IMS Health Real-World Data Medical Claims database was used to identify treatment-naive patients receiving ranibizumab intravitreal injections or dexamethasone intravitreal implants between June 2010 and February 2014 who had 12 months of follow-up data. The primary outcome measure was the mean number of all ophthalmology visits for the two drugs in patients with CRVO and BRVO. Secondary outcome measures included a comparison of treatment visits, non-treatment visits, and time intervals between visits.

Results Overall, 2822 patients received ranibizumab injections (CRVO, 1178; BRVO, 1644) and 365 received dexamethasone implants (CRVO, 191; BRVO, 174). The mean number (SD) of all ophthalmology visits was higher for patients receiving ranibizumab injections than for those receiving dexamethasone implants (CRVO: 7.2 (3.6) vs 6.2 (3.1), $P<0.001$; BRVO: 7.1 (3.4) vs 6.3 (3.1), $P=0.016)$.

Conclusions Patients with RVO receiving ranibizumab injections had a mean of approximately one more visit to their ophthalmologist in the first $\mathbf{1 2}$ months of treatment than those treated with dexamethasone implants. The visit burden is
\end{abstract}

therefore not substantially different and physicians should focus on the clinical benefits of these drugs when evaluating treatment options for RVO.

Eye (2017) 31, 551-559; doi:10.1038/eye.2016.269; published online 2 December 2016

\section{Introduction}

Macular oedema (MO) secondary to retinal vein occlusion (RVO) is the second most common cause of retinal vascular disease, and can result in significant vision loss. ${ }^{1,2}$ The pathogenesis of $\mathrm{RVO}$ is multifactorial, but the retinal veins typically become obstructed owing to vascular clots, external vascular compression or vessel wall pathology. ${ }^{3}$ The obstruction can occur in either the central retinal vein or branches of the retinal veins, and is referred to as central RVO (CRVO) and branch RVO (BRVO), respectively. ${ }^{2}$ BRVO is more common than CRVO, with prevalence estimates of 4.42 per $1000(95 \%$ confidence interval (CI): $3.65-5.19)$ for BRVO compared with 0.80 per 1000 (95\% CI: $0.61-0.99)$ for CRVO. ${ }^{4}$ Occlusion of the retinal veins causes an increase in capillary pressure, which induces expression of vascular endothelial growth factor (VEGF) and interleukin-6.5,6 This causes a breakdown of the blood-retinal barrier and increases vascular permeability, ${ }^{6}$ leading to complications such as $\mathrm{MO}$ and retinal ischemia, ultimately resulting in vision loss. ${ }^{3}$ Until recently, the standard of care for MO secondary to RVO comprised corticosteroids (CRVO and BRVO) and laser photocoagulation (BRVO only); ${ }^{7}$ however, anti-VEGF agents provide an additional therapeutic option for both BRVO and CRVO. ${ }^{7-11}$

Ranibizumab (Lucentis) is a humanized, VEGF antibody fragment that binds and
${ }^{1}$ Centre Ophtalmologique d'Imagerie et de Laser, Paris, France, Department of Ophthalmology, Hôpital Avicenne, Assistance Publique, Hôpitaux de Paris and Paris 13 University, Bobigny, France

${ }^{2}$ Department of Ophthalmology, Pasteur II University Hospital, Nice, France

${ }^{3}$ Novartis Pharma AG, Basel, Switzerland

${ }^{4}$ Novartis Ireland Limited, Dublin, Ireland

${ }^{5}$ Real-World Evidence Solutions, IMS Health, London, UK

${ }^{6}$ Department of Ophthalmology, Careggi University Hospital, Florence, Italy

Correspondence: S Nghiem-Buffet, Centre Ophtalmologique d'Imagerie et de Laser, 11 rue Antoine Bourdelle, 75015 Paris, France Tel: +33 1428494 00; Fax: +33 142849409 . E-mail: buffet.nghiem@free.fr

Received: 25 January 2016 Accepted in revised form: 14 October 2016 Published online: 2 December 2016 
neutralizes multiple isoforms of VEGF. ${ }^{11}$ The efficacy and safety of ranibizumab intravitreal injection were assessed in two randomized, phase three clinical trials (BRAVO and CRUISE, ClinicalTrial.gov Identifiers NCT00486018 and NCT00485836, respectively), in which patients with BRVO and CRVO were administered the study drug once per month for 6 months and compared with sham-treated individuals. ${ }^{12,13}$ In both studies, a higher proportion of patients treated with ranibizumab experienced improvements in vision relative to baseline than individuals who received sham injections $(P<0.0001)$, as assessed by a gain of 15 or more letters in best corrected visual acuity (BCVA). This was accompanied by a higher mean (95\% confidence interval) change in BCVA letter score from baseline in patients treated with ranibizumab than in those who received sham injections (18.3 (16.020.6) vs 7.3 (5.1-9.5), respectively). ${ }^{12,13}$ These

improvements were maintained for up to 12 months. ${ }^{14,15}$ Ocular adverse events also occurred at a lower frequency in the ranibizumab group than in the sham-treated group. ${ }^{12,13}$ As a consequence, ranibizumab was approved for the treatment of MO secondary to RVO by the US Food and Drug Administration (FDA) in 2010 (ref. 16) and by the European Medicines Agency (EMA) in 2011. ${ }^{17}$ Clinical trial data were later supported by a series of realworld evidence studies, which provided further evidence supporting the effectiveness and safety profile of ranibizumab for the treatment of patients with retinal disease. ${ }^{18-20}$ The recommended dose of ranibizumab in patients with CRVO and BRVO is $0.5 \mathrm{mg}(0.05 \mathrm{ml}$ solution) administered as a single intravitreal injection once per month in the USA. ${ }^{21}$

Dexamethasone, a water-soluble corticosteroid, has also been shown to be efficacious in the treatment of patients with CRVO and BRVO. ${ }^{22}$ Dexamethasone is delivered directly to the vitreous cavity by an intravitreal implant (Ozurdex). ${ }^{23}$ A sham-controlled clinical trial (GENEVA, ClinicalTrial.gov Identifier NCT01660802) of dexamethasone implant demonstrated significant improvements in BCVA scores and in the percentage of eyes with an improvement of at least 15 letters in BCVA in patients with CRVO and BRVO, compared with shamtreated patients, from day 30 to day 90 after treatment initiation $(P<0.001) .{ }^{22}$ However, results from this trial have also shown that the incidence of ocular adverse events was significantly higher in patients treated with dexamethasone implant than in sham-treated individuals. ${ }^{22,24}$

A recent study by Lam et al provided evidence that dexamethasone implant also provides real-world anatomical and functional improvements in patients with MO associated with retinal disease. ${ }^{25}$ Furthermore, this study did not identify any new safety concerns. ${ }^{25}$ Dexamethasone implant was approved for the treatment of MO associated with RVO by the US FDA in 2009 (ref. 26 and by the EMA in 2010. ${ }^{27}$ The reported re-treatment period for dexamethasone implant is every 6 months, ${ }^{26}$ and there is limited information available on shorter re-treatment intervals. ${ }^{23}$ However, from a retrospective, consecutive case series of 49 patients with MO secondary to RVO, dosing every 6 months was found to be insufficient, and improved results were achieved with an 'as-needed' re-treatment protocol. ${ }^{28}$ Similarly, a recent prospective study of 35 patients indicated that the optimal time for re-treatment for most patients with $\mathrm{ME}$ secondary to RVO is $<6$ months after the first dexamethasone treatment. ${ }^{29}$

Head-to-head clinical trial results ${ }^{30,31}$ and indirect, retrospective analyses of clinical trial data ${ }^{32-34}$ demonstrate improved efficacy and safety for patients treated with ranibizumab compared with those treated with dexamethasone implant. It has been suggested, however, that differences in protocol and dosing regimens in these retrospective studies could have led to potential bias (eg, differences in inclusion/exclusion criteria and differences in patient characteristics at baseline). ${ }^{11}$ Therefore, the findings from the aforementioned studies need to be interpreted with caution. Furthermore, anti-VEGF intraocular injections may be associated with a potential increase in the rates of systemic adverse events in patients receiving these treatments. ${ }^{35}$

In light of different treatment regimens and dosing guidelines, the primary objective of this study was to compare the mean number of all ophthalmology visits for patients receiving ranibizumab intravitreal injection or dexamethasone intravitreal implant for CRVO or BRVO during the first 12 months after treatment initiation.

\section{Materials and methods}

\section{Study design}

This was a retrospective study using insurance claims data entered by physicians in the USA into the IMS Health Real-World Data (RWD) Medical Claims database (managed by IMS Health, Plymouth Meeting, PA, USA). Information about the database is published elsewhere. ${ }^{36}$ Patient data used in this study were anonymized to comply with the Health Insurance Portability and Accountability Act (HIPAA). The study was also designed and implemented in accordance with the Guidelines for Good Pharmacoepidemiology Practice (GPP) of the International Society for Pharmacoepidemiology (ISPE) and the Strengthening the Reporting of Observational Studies in Epidemiology (STROBE) guidelines. ${ }^{37}$ 


\section{Study population}

Patients were included in the study if they had received ranibizumab intravitreal injection or dexamethasone intravitreal implant and had a concomitant diagnosis of CRVO or BRVO between June 2010 and February 2014. Diagnoses were defined using the International Classification of Diseases, Ninth Revision, Clinical Modification (ICD-9-CM); ${ }^{38}$ CRVO was defined as ICD-9 362.35 and BRVO as ICD-9 362.36. The first claim for either ranibizumab injection or dexamethasone implant was defined as the patient's index date. Eligible patients were subdivided according to the index diagnosis (CRVO or BRVO). Data from September 2009 to January 2015 were extracted to ensure at least a 9-month pre-index period and a 12-month follow-up period. Patients were excluded if any of the following criteria were met:

(i) patient was younger than 18 years of age at index date; (ii) physician administering the index medicine did not submit monthly medical claims during the 9-month preindex period and during the 12-month post-index period; (iii) patient received one or more anti-VEGF injections or dexamethasone implants during the 9-month pre-index period; (iv) patient received one or more anti-VEGF injections or dexamethasone implants other than index treatment during the 12-month follow-up period in either eye; (v) patient did not have a claim record after the end of the 12-month follow-up period. The selection process and attrition data for the study population (with 12-months of follow-up data) are shown in Figure 1.

\section{Outcome measures}

The primary outcome measure was the mean number of all ophthalmology visits during the 12-month post-index period for the ranibizumab injection and dexamethasone implant groups (with restriction to index provider). The secondary outcome measures were: (i) the number of treatment visits; (ii) the number of non-treatment visits; and (iii) the differences in time intervals (days) between visits for: all visits, treatment visits and non-treatment visits during the 12-month post-index period. A treatment visit was defined as a visit at which the index treatment was administered during the 12-month follow-up period.

All outcome measures for the two treatment groups were compared separately for patients with CRVO and for those with BRVO. In addition, several sensitivity analyses were performed. First, unilateral and bilateral treatments were evaluated to examine treatment patterns at eye level. Second, the frequency of all ophthalmology visits, treatment visits and non-treatment visits was assessed with and without restriction to the index provider. Finally, the frequency of these visits was assessed in a subgroup of patients with 24 months of follow-up data (patient selection and attrition data for the subgroup are shown in Supplementary Figure 1).

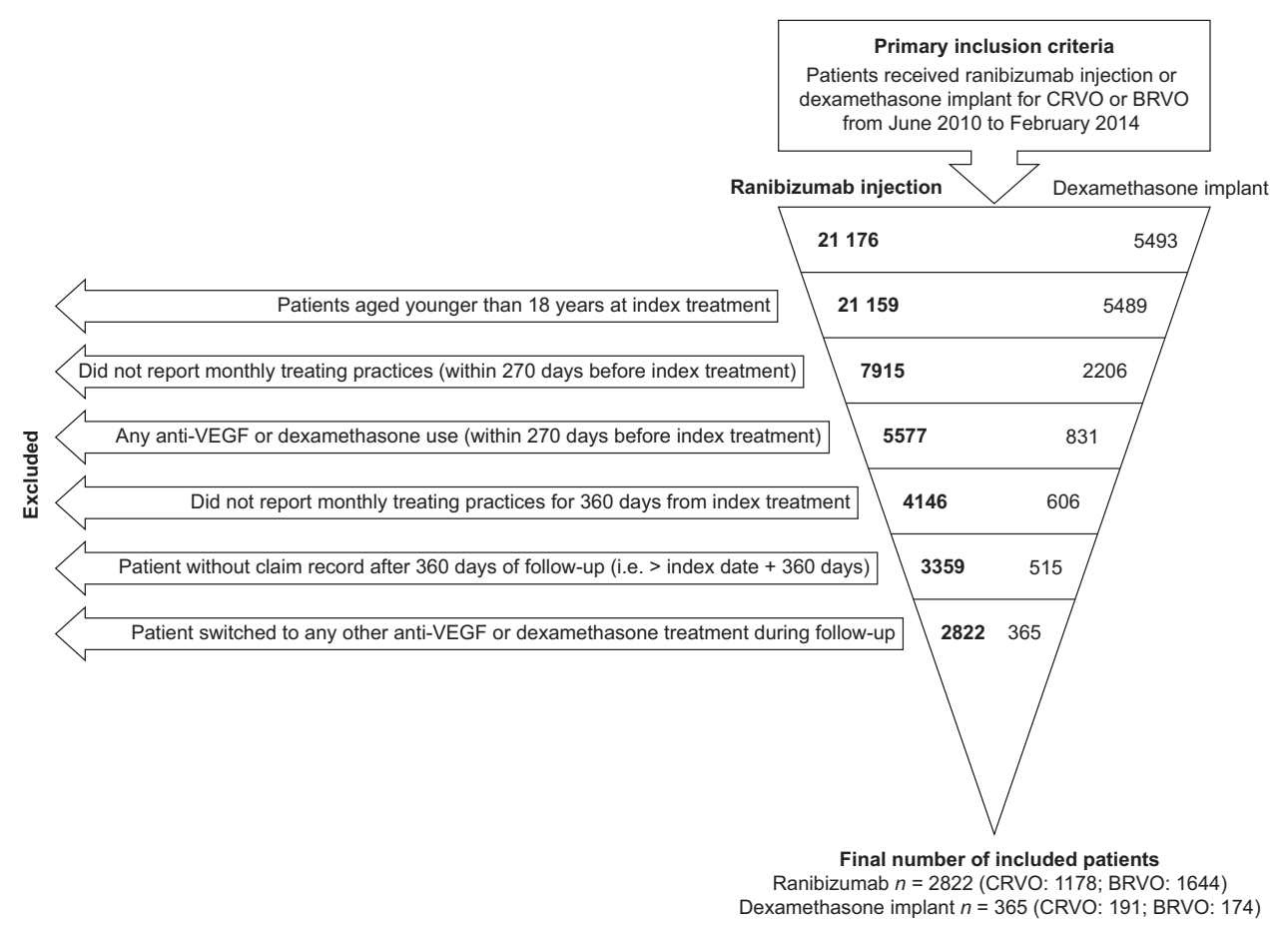

Figure 1 Selection process and attrition of patient groups for patients who had 12 months of follow-up data. BRVO, branch retinal vein occlusion; CRVO, central retinal vein occlusion; VEGF, vascular endothelial growth factor. 


\section{Statistical analysis}

The mean (standard deviation (SD)) was used for continuous variables and $n(\%)$ for categorical variables to describe patient characteristics at baseline and outcome measures between treatment groups. Differences in baseline characteristics between treatment groups were compared using the Student's $t$-test, Wilcoxon signedrank test or chi-squared test, as appropriate. The generalized linear model (GENMOD procedure) using negative binomial distribution compared the number of visits and intervals between treatment groups, adjusting for patients' baseline characteristics. A $P$ value of $<0.05$ was considered statistically significant. Statistical analyses were performed with SAS 9.4 (SAS Institute Inc., Cary, NC, USA).

\section{Results}

\section{Demographics and baseline characteristics}

In total, 2822 patients receiving ranibizumab injections (CRVO, $n=1178$; BRVO, $n=1644$ ) and 365 patients receiving dexamethasone implants $(\mathrm{CRVO}, n=191$; BRVO, $n=174$ ) were identified with at least 12 months of follow-up data (Figure 1). Demographics and baseline characteristics for the 12-month group are shown in Table 1. Patients who received ranibizumab injections were older than those who received dexamethasone implants in the CRVO group (mean age: 72.8 years vs 68.8 years; $P<0.0001)$, but were of a similar age in the BRVO group (mean age: 73.3 years $v s 72.7$ years; $P=0.525$ ).
Patients with CRVO in the ranibizumab injection group had lower mean Charlson-Deyo Comorbidity Index scores (SD) than those in the dexamethasone implant group (0.7 (1.3) vs 1.0 (1.4), $P=0.011)$, but the comorbidity scores were similar for patients with BRVO (0.6 (1.1) vs 0.6 (1.0), $P=0.924)$. There were also some regional differences in treatment patterns observed between the treatment groups (Table 1).

\section{Overall number of ophthalmology visits}

In the first 12 months after treatment initiation, the mean number of all ophthalmology visits for patients with CRVO (SD) was 7.2 (3.6) for patients who received ranibizumab injections and 6.2 (3.1) for those who received dexamethasone implants $(P<0.001)$. The mean number of all ophthalmology visits was also higher for patients with BRVO who received ranibizumab injections than for those who received dexamethasone implants (7.1 (3.4) vs 6.3 (3.1), $P=0.016$; Figure 2). The distribution of all ophthalmology visits in the first 12 months is presented in Figure 3.

\section{Visits with and without treatment and visit intervals}

The mean number (SD) of treatment visits was higher for patients in the ranibizumab injection group than for those in the dexamethasone implant group for CRVO (4.1 (2.7) vs 1.8 (1.0), $P<0.0001)$ and for BRVO (3.9 (2.6) vs 1.7 (1.0), $P<0.0001)$. In contrast, the mean number of non-treatment visits was higher in the dexamethasone

Table 1 Demographics and baseline characteristics for patients with CRVO or BRVO treated with ranibizumab injection or dexamethasone implant who had 12 months of follow-up data

\begin{tabular}{|c|c|c|c|c|c|c|}
\hline \multirow[t]{2}{*}{ Characteristic } & \multicolumn{3}{|c|}{ CRVO $(n=1369)$} & \multicolumn{3}{|c|}{$B R V O(n=1818)$} \\
\hline & $\begin{array}{c}\text { Ranibizumab } \\
\text { injection } \\
(n=1178)\end{array}$ & $\begin{array}{c}\text { Dexamethasone } \\
\text { implant } \\
(n=191)\end{array}$ & P-value & $\begin{array}{c}\text { Ranibizumab } \\
\text { injection } \\
(n=1644)\end{array}$ & $\begin{array}{c}\text { Dexamethasone } \\
\text { implant } \\
(n=174)\end{array}$ & P-value \\
\hline Age, mean, years (SD) & $72.8(11.5)$ & $68.8(11.8)$ & $<0.0001$ & $73.3(10.2)$ & $72.7(10.5)$ & 0.525 \\
\hline Female, $n(\%)$ & $623(52.9)$ & $98(51.3)$ & 0.686 & $948(57.7)$ & $89(51.2)$ & 0.099 \\
\hline Charlson-Deyo Comorbidity Index, mean (SD) & $0.7(1.3)$ & $1.0(1.4)$ & 0.011 & $0.6(1.1)$ & $0.6(1.0)$ & 0.924 \\
\hline \multicolumn{7}{|l|}{ Payer type, $n(\%)$} \\
\hline Commercial & $382(32.4)$ & $83(43.5)$ & $<0.0001$ & 512 (31.1) & 66 (37.9) & 0.113 \\
\hline Medicare & $781(66.3)$ & $100(52.4)$ & & $1116(67.9)$ & $108(62.1)$ & \\
\hline Medicaid & $15(1.3)$ & $8(4.2)$ & & $16(1.0)$ & $0(0.0)$ & \\
\hline \multicolumn{7}{|l|}{ US geographical region, $n(\%)$} \\
\hline Midwest & $177(15.0)$ & $19(9.9)$ & 0.034 & $290(17.6)$ & $31(17.8)$ & $<0.0001$ \\
\hline Northeast & $333(28.3)$ & $61(31.9)$ & & 485 (29.5) & $46(26.4)$ & \\
\hline West & $516(43.8)$ & 75 (39.3) & & $689(41.9)$ & 55 (31.6) & \\
\hline South & 152 (12.9) & $36(18.8)$ & & $180(10.9)$ & $42(24.1)$ & \\
\hline
\end{tabular}

Abbreviations: BRVO, branch retinal vein occlusion; CRVO, central retinal vein occlusion; SD, standard deviation. 

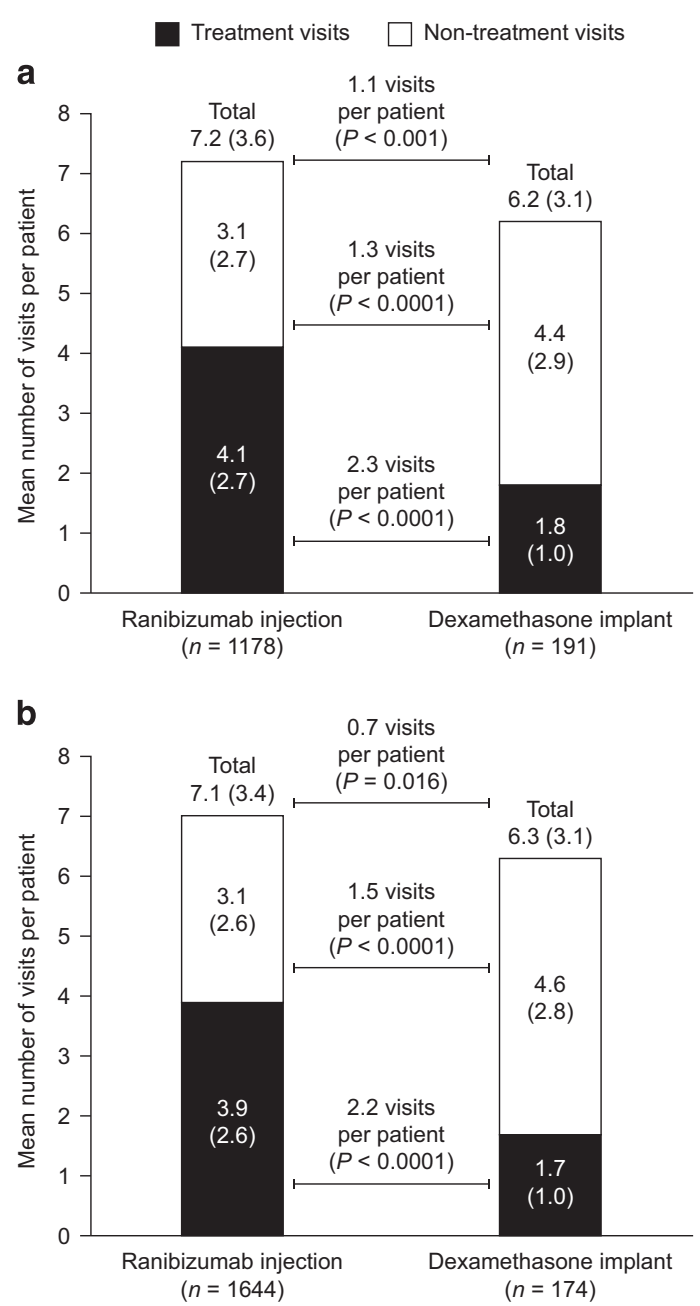

Figure 2 Mean number (SD) of all ophthalmology visits in the first 12 months after treatment initiation for patients with (a) CRVO or (b) BRVO treated with ranibizumab injection or dexamethasone implant. Differences between treatment groups were compared after adjusting for baseline characteristics (age, sex, Charlson-Deyo Comorbidity Index, health plan type, and US geographical region). BRVO, branch retinal vein occlusion; $\mathrm{CRVO}$, central retinal vein occlusion; $\mathrm{SD}$, standard deviation.

implant group than in the ranibizumab injection group for patients with CRVO (3.1 (2.7) vs 4.4 (2.9), $P<0.0001)$ and with BRVO (3.1 (2.6) vs 4.6 (2.8), $P<0.0001$; Figure 2).

The time interval between these visits was also recorded (Table 2). Overall, the mean time interval (in days) was shorter for all ophthalmology visits and treatment visits for patients receiving ranibizumab injections than for those who received dexamethasone implants for CRVO $(P<0.0001)$ and BRVO $(P<0.0001)$. The mean time interval between non-treatment visits was similar for both treatments for patients with BRVO $(P=0.702)$, but was shorter for patients with CRVO $(P=0.009)$ who received dexamethasone implants than for those who received ranibizumab injections.
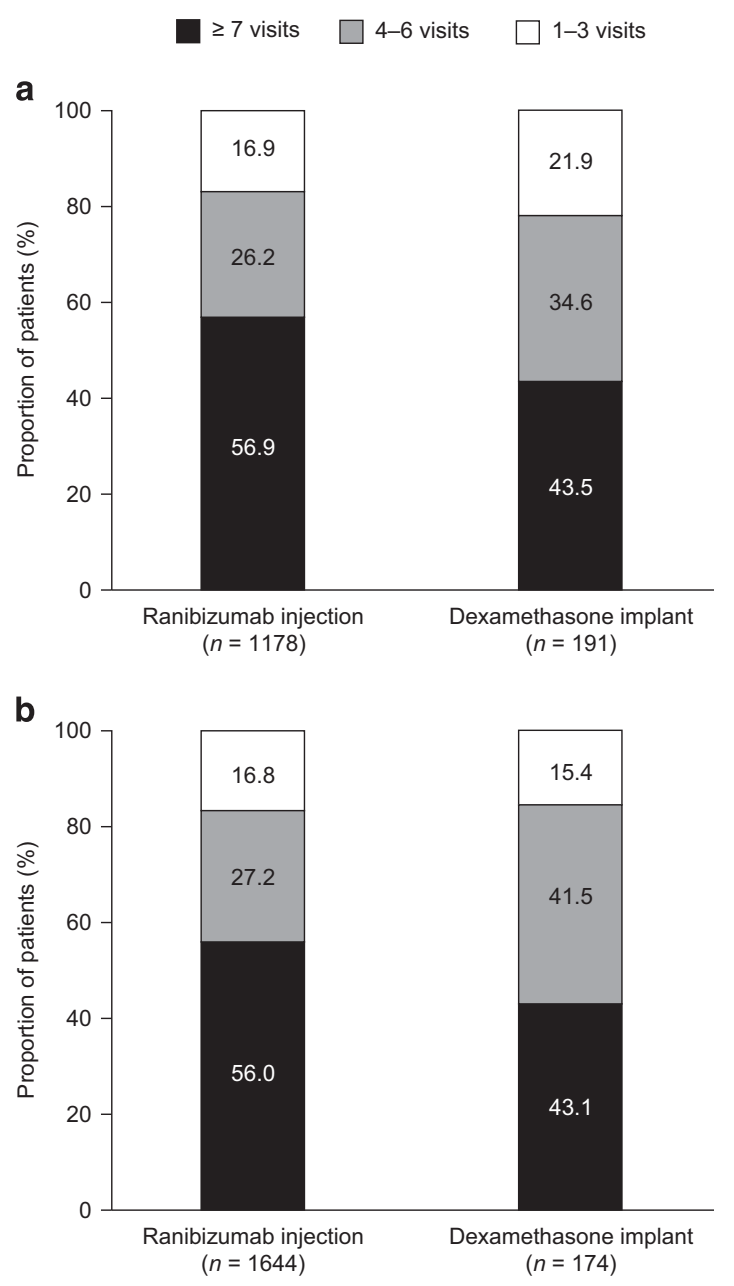

Figure 3 Distribution of the number of all ophthalmology visits in the first 12 months after treatment initiation for patients with (a) CRVO or (b) BRVO treated with ranibizumab injection or dexamethasone implant. BRVO, branch retinal vein occlusion; CRVO, central retinal vein occlusion.

\section{Sensitivity analyses}

In both treatment groups, most patients (over 96\%) received unilateral treatment (treatment to one eye only). The mean number of unilateral treatment administrations (SD) to the index eye (eye receiving treatment at index date) was higher during the first 12 months of treatment for patients who received ranibizumab injections than for those who received dexamethasone implants (CRVO: 4.0 (2.6) vs 1.6 (0.9), $P<0.0001$; BRVO: 3.8 (2.6) vs 1.6 (0.9), $P<0.0001)$.

When the restriction to index provider was relaxed, the number of all ophthalmology visits for patients with 12 months of follow-up data remained higher in the group receiving ranibizumab injections than in the group receiving dexamethasone implants (CRVO: 8.4 (4.4) vs 7.1 (3.8), $P<0.0001$; BRVO: 8.0 (3.8) vs 7.5 (3.8), $P=0.0716$ ). 
Table 2 Mean time intervals between visits for patients with CRVO or BRVO treated with ranibizumab injection or dexamethasone implant who had 12 months of follow-up data

\begin{tabular}{|c|c|c|c|c|c|c|}
\hline \multirow{2}{*}{$\begin{array}{l}\text { Time interval between } \\
\text { visits, days }\end{array}$} & \multicolumn{3}{|c|}{ CRVO $(n=1369)$} & \multicolumn{3}{|c|}{$B R V O(n=1818)$} \\
\hline & $\begin{array}{c}\text { Ranibizumab } \\
\text { injection } \\
(n=1178)\end{array}$ & $\begin{array}{c}\text { Dexamethasone } \\
\text { implant } \\
(n=191)\end{array}$ & P-value & $\begin{array}{c}\text { Ranibizumab } \\
\text { injection } \\
(n=1644)\end{array}$ & $\begin{array}{c}\text { Dexamethasone } \\
\text { implant } \\
(n=174)\end{array}$ & P-value \\
\hline All ophthalmology visits & $46.8(26.0)$ & $55.3(38.2)$ & $<0.0001$ & $47.8(25.2)$ & $59.6(33.2)$ & $<0.0001$ \\
\hline Treatment visits & $61.7(42.9)$ & $159.0(71.1)$ & $<0.0001$ & $63.2(43.3)$ & $162.1(72.2)$ & $<0.0001$ \\
\hline Non-treatment visits & $77.4(50.4)$ & $66.9(38.9)$ & 0.009 & $75.8(43.7)$ & $74.6(44.1)$ & 0.702 \\
\hline
\end{tabular}

Abbreviations: BRVO, branch retinal vein occlusion; CRVO, central retinal vein occlusion; SD, standard deviation.

Data are shown as mean (SD).

When applying the 24-month post-index restrictions (Supplementary Figure 1), a subset of patients who received either ranibizumab injections $(n=1252)$ or dexamethasone implants $(n=160)$ were identified. Baseline characteristics for these patients have been included as supplementary information (Supplementary Table 1). For this subpopulation, the mean number (SD) of all ophthalmology visits in the first 24 months after treatment initiation for those who received ranibizumab injections was higher than for those who received dexamethasone implants (CRVO: 11.0 (7.0) vs 9.3 (5.8), $P=0.115$; BRVO: 10.5 (6.3) vs 9.0 (5.6), $P=0.038$ ). The results for the majority of secondary outcome measures were similar to those for the main study findings and are presented in Supplementary Table 2.

\section{Discussion}

This non-interventional, patient-level claims database study is the first to compare treatment patterns between ranibizumab injection and dexamethasone implant for patients with CRVO or BRVO in the USA. The main finding of this study is that individuals with CRVO or BRVO who received ranibizumab injections had a mean of approximately one more visit to their ophthalmologist during the first 12 months of treatment than patients treated with dexamethasone implant (all ophthalmology visits). The mean number of treatment visits in the first 12 months was also higher in patients who received ranibizumab injection than in those who received dexamethasone implant; however, the mean number of non-treatment visits was higher in patients who received dexamethasone implant than in those who received ranibizumab. The majority of these findings were similar in patients with 24 months of follow-up data.

In the USA, it is recommended that ranibizumab is administered as a monthly intravitreal injection $(0.5 \mathrm{mg})$ for $\mathrm{MO}$ secondary to RVO, although this may be changed to a less effective regimen ${ }^{21}$ (one injection every 3 months after the first four injections), if monthly injections are not feasible. ${ }^{21}$ In Europe, treatment intervals with ranibizumab may also be gradually extended. ${ }^{39}$ Monthly injections may not be feasible for some patients, owing to the perceived inconvenience of frequent treatment visits and potential transportation issues to and from the clinic or hospital. ${ }^{40}$ These factors may influence a clinician's choice of treatment. In contrast, dexamethasone is administered as an implant that releases a dose of $0.7 \mathrm{mg}$ over several months (reportedly over a 6-month period). ${ }^{26,41}$ The results presented in this study indicate that patients do not receive ranibizumab at the recommended interval for the first 12 months after treatment initiation. This could be because of improvements in visual acuity in the first 6 months, ${ }^{12,13}$ or because of the perceived inconvenience for patients. ${ }^{40}$

For patients who received dexamethasone implant, the mean number of all ophthalmology visits was higher than expected during the first 12 months of treatment, even though the implant is a slow-release treatment and, as per the US label, patients require substantially fewer physician visits than those treated with monthly ranibizumab. This is accounted for by the increased number of non-treatment visits by patients who received dexamethasone implant, which was also observed 24 months after treatment initiation. One potential explanation for this could be the increased frequency of ocular adverse events (cataracts and elevated intraocular pressure) associated with dexamethasone treatment. In clinical trials, the frequency of ocular adverse events was higher in dexamethasone-treated individuals than in those who received sham injections, although the increase in intraocular pressure was usually transient. ${ }^{24,34,41}$ Physicians are thus more likely to screen for such adverse events in patients treated with dexamethasone. Therefore, it could be argued that the visit burden of both treatments is not likely to be substantially different in routine clinical practice.

One strength of this study is that it utilized large-scale, routinely-collected population data from one of the largest medical claims databases in the USA. Of claims submitted to the IMS Health RWD Medical Claims database, $95 \%$ are available to view within 3 weeks of the 
submission date. The sensitivity analyses support the main findings of this study, and demonstrate that the results presented here are not confounded by the restriction to index provider. Furthermore, the data presented on the number of ranibizumab injections received by patients with CRVO are similar to figures published elsewhere, ${ }^{36}$ demonstrating the reproducibility of the methods used. This study has also highlighted that the manner in which therapeutic agents are used in routine clinical practice may differ significantly from the label recommendations, emphasizing the importance of real-world evidence studies.

The nature of the study has led to several limitations. First, some of the baseline characteristics for patients treated with ranibizumab injections differed from those treated with dexamethasone implant and could have therefore introduced unknown bias. For example, patients with CRVO who received ranibizumab were older than those who received dexamethasone implant $(P<0.001)$. This result was surprising because dexamethasone treatment is associated with an increased risk of cataract development, ${ }^{42}$ and is therefore usually prescribed to older patients for whom the development of cataracts is intrinsically more common. ${ }^{43}$ This side effect could therefore be viewed as less serious in older patients receiving dexamethasone treatment. This study also used physician-entered claims codes to assess treatment patterns. Therefore, misclassification by physicians was a possibility, although this is likely to have affected both groups equally. As the database is used for billing and reimbursement purposes, and not specifically for research use, it may not capture all information, including the purpose of each patient's visit to their physician. We were therefore unable to assess the effect of treatment frequency on improvements or worsening in clinical outcomes. Finally, this study examined treatment patterns for patients in the USA, and the results may therefore not be applicable to other countries.

In conclusion, this study is the first to compare treatment patterns between ranibizumab injection and dexamethasone implant in patients with CRVO and BRVO in the USA, using a large physician-entered medical claims database. In the first 12 months of treatment, patients who received ranibizumab intravitreal injection had a mean of approximately one more visit to their ophthalmologist than those who received dexamethasone implant, which was driven primarily by the high number of non-treatment visits for patients in the dexamethasone implant group. The visit burden of these drugs is thus not likely to be substantially different in routine clinical practice. Physicians should therefore consider the risk-benefit profile of anti-VEGF and corticosteroid implant therapies when deciding on the appropriate treatment for patients with RVO. Further long-term analyses on larger patient populations in different countries combined with comparisons of ocular safety profiles would help to inform the development of global care pathways further for patients with CRVO and BRVO.

\section{Summary}

\section{What was known before}

- Ranibizumab intravitreal injection and dexamethasone intravitreal implant have been shown to be effective in the treatment of macular oedema secondary to retinal vein occlusion (RVO) (central RVO (CRVO) and branch RVO (BRVO)).

- Ranibizumab injection is recommended to be administered once per month as per the US label. This is in contrast to the dexamethasone implant, which slowly releases the dose over a series of months.

What this study adds

- This study used US patient-level medical claims data to compare real-world treatment patterns of ranibizumab injection and dexamethasone implant in patients with CRVO or BRVO.

- The findings demonstrate that patients with CRVO or BRVO receiving ranibizumab injections had a mean of approximately one more visit to their ophthalmologist in the first 12 months of treatment than patients receiving dexamethasone implant.

- The visit burden of ranibizumab injections and dexamethasone implants is therefore not likely to be substantially different in routine clinical practice in the USA.

\section{Conflict of interest}

Sylvia Nghiem-Buffet and Stéphanie Baillif are consultants for Allergan, Bayer and Novartis. Stephane Regnier is an employee of Novartis Pharma AG, Basel, Switzerland. Adrian Skelly is an employee of Novartis Ireland Limited, Dublin, Ireland. Ning Yu is an employee of IMS Health, London, UK, funded by Novartis to perform the statistical analyses for this study, but was not involved in the collection of the dataset or in gaining access to it for the purposes of the study. Andrea Sodi is a member of the Novartis RVO advisory board.

\section{Author contributions}

All authors were involved in the design of this study, data acquisition, and/or played an important role in the interpretation of the study results. All authors have been involved in the development and revision of the manuscript and have approved the final version.

\section{Acknowledgements}

This study was funded by Novartis Pharma AG. Medical writing support was provided by Luci Witcomb, 
PhD, of PharmaGenesis London, UK, and funded by Novartis Pharma AG.

\section{References}

1 McIntosh RL, Rogers SL, Lim L, Cheung N, Wang JJ, Mitchell $\mathrm{P}$ et al. Natural history of central retinal vein occlusion: an evidence-based systematic review. Ophthalmology 2010; 117(6): 1113-1123.

2 Wong TY, Scott IU. Clinical practice. Retinal-vein occlusion. New Eng J Med 2010; 363(22): 2135-2144.

3 Rehak J, Rehak M. Branch retinal vein occlusion: pathogenesis, visual prognosis, and treatment modalities. Curr Eye Res 2008; 33: 111-131.

4 Rogers S, McIntosh RL, Cheung N, Lim L, Wang JJ, Mitchell $\mathrm{P}$ et al. The prevalence of retinal vein occlusion: pooled data from population studies from the United States, Europe, Asia, and Australia. Ophthalmology 2010; 117(2): 313-319.

5 Yoshimura T, Sonoda KH, Sugahara M, Mochizuki Y, Enaida H, Oshima $Y$ et al. Comprehensive analysis of inflammatory immune mediators in vitreoretinal diseases. PlOS One 2009; 4(12): e8158.

6 Noma H, Funatsu H, Mimura T, Harino S, Sone T, Hori S. Increase of vascular endothelial growth factor and interleukin-6 in the aqueous humour of patients with macular oedema and central retinal vein occlusion. Acta Ophthalmol 2010; 88(6): 646-651.

7 Gerding H, Mones J, Tadayoni R, Boscia F, Pearce I, Priglinger S. Ranibizumab in retinal vein occlusion: treatment recommendations by an expert panel. Br J Ophthalmol 2015; 99(3): 297-304.

8 Ip MS, Scott IU, VanVeldhuisen PC, Oden NL, Blodi BA, Fisher $\mathrm{M}$ et al. A randomized trial comparing the efficacy and safety of intravitreal triamcinolone with observation to treat vision loss associated with macular edema secondary to central retinal vein occlusion: the Standard Care vs Corticosteroid for Retinal Vein Occlusion (SCORE) study report 5. Arch Ophthalmol 2009; 127(9): 1101-1114.

9 National Institute for Health and Care Excellence. TA 283. Ranibizumab for treating visual impairment caused by macular oedema secondary to retinal vein occlusion. Accessed on November 2015. https:/ / www.nice.org.uk/ guidance/ta283.

10 Evaluation of grid pattern photocoagulation for macular edema in central vein occlusion. The Central Vein Occlusion Study Group M report. Ophthamology 1995; 102(10): 1425-1433.

11 Glanville J, Patterson J, McCool R, Ferreira A, Gairy K, Pearce I. Efficacy and safety of widely used treatments for macular oedema secondary to retinal vein occlusion: a systematic review. BMC Ophthalmol 2014; 14: 7.

12 Brown DM, Campochiaro PA, Singh RP, Li Z, Gray S, Saroj $\mathrm{N}$ et al. Ranibizumab for macular edema following central retinal vein occlusion: six-month primary end point results of a phase III study. Ophthalmology 2010; 117(6): 1124-1133.

13 Campochiaro PA, Heier JS, Feiner L, Gray S, Saroj N, Rundle AC et al. Ranibizumab for macular edema following branch retinal vein occlusion: six-month primary end point results of a phase III study. Ophthalmology 2010; 117(6): 1102-1112.
14 Brown DM, Campochiaro PA, Bhisitkul RB, Ho AC, Gray S, Saroj $\mathrm{N}$ et al. Sustained benefits from ranibizumab for macular edema following branch retinal vein occlusion: 12-month outcomes of a phase III study. Ophthalmology 2011; 118(8): 1594-1602.

15 Campochiaro PA, Brown DM, Awh CC, Lee SY, Gray S, Saroj $\mathrm{N}$ et al. Sustained benefits from ranibizumab for macular edema following central retinal vein occlusion: twelve-month outcomes of a phase III study. Ophthalmology 2011; 118(10): 2041-2049.

16 Genentech. FDA approves Lucentis (ranibizumab injection) for the treatment of macular edema following retinal vein occlusion. Accessed on November 2015. http://www. gene.com/media/press-releases/12827/2010-06-22/fdaapproves-lucentis-ranibizumab-inject.

17 European Medicines Agency. Committee for Medicinal Products for Human use. Summary of opinion. Lucentis (ranibizumab). Accessed on November 2015.; EMA/CHMP/ 221398/2011. http://www.ema.europa.eu/docs/en_GB/ document_library/Summary_of_opinion/human/000715/ WC500103866.pdf.

18 Rakic JM, Leys A, Brie H, Denhaerynck K, Pacheco C, Vancayzeele $\mathrm{S}$ et al. Real-world variability in ranibizumab treatment and associated clinical, quality of life, and safety outcomes over 24 months in patients with neovascular agerelated macular degeneration: the HELIOS study. Clin Ophthalmol 2013; 7: 1849-1858.

19 Rezar S, Eibenberger K, Buhl W, Georgopoulos M, Schmidt-Erfurth U, Sacu $S$ et al. Anti-VEGF treatment in branch retinal vein occlusion: a real-world experience over 4 years. Acta Ophthalmol 2015; 93: 719-725.

20 Holz FG, Bandello F, Gillies M, Mitchell P, Osborne A, Sheidow $\mathrm{T}$ et al. Safety of ranibizumab in routine clinical practice: 1-year retrospective pooled analysis of four European neovascular AMD registries within the LUMINOUS programme. Br J Ophthalmol 2013; 97(9): 1161-1167.

21 Food and Drug Administration. Highlights of prescribing information. Lucentis (ranibizumab injection). Accessed on November 2015. http:/ / www.accessdata.fda.gov/ drugsatfda_docs/label/2006/125156lbl.pdf.

22 Haller JA, Bandello F, Belfort Jr R, Blumenkranz MS, Gillies M, Heier J et al. Randomized, sham-controlled trial of dexamethasone intravitreal implant in patients with macular edema due to retinal vein occlusion. Ophthalmology 2010; 117(6): 1134-1146.

23 European Medicines Agency. Ozurdex (dexamethasone). Summary of product characteristics. Accessed on November 2015. http://www.ema.europa.eu/docs/en_GB/ document_library/EPAR__Product_Information/human/ 001140/WC500095499.pdf.

24 Haller JA, Bandello F, Belfort Jr R, Blumenkranz MS, Gillies M, Heier J et al. Dexamethasone intravitreal implant in patients with macular edema related to branch or central retinal vein occlusion twelve-month study results. Ophthalmology 2011; 118(12): 2453-2460.

25 Lam WC, Albiani DA, Yoganathan P, Chen JC, Kherani A, Maberley DA et al. Real-world assessment of intravitreal dexamethasone implant $(0.7 \mathrm{mg})$ in patients with macular edema: the CHROME study. Clin Ophthalmol 2015; 9: 1255-1268.

26 Christoforidis JB, Chang S, Jiang A, Wang J, Cebulla CM. Intravitreal devices for the treatment of vitreous inflammation. Mediators Inflamm 2012; 2012: 1-8. 
27 European Medicines Agency. Committee for Medicinal Products for Human use. Summary of opinion. Ozurdex (dexamethasone). Accessed on November 2015; EMA/ CHMP/316582/2010. http:/ / www.ema.europa.eu/docs/ en_GB/document_library/Summary_of_opinion_-_Initial_ authorisation/human/001140/WC500090817.pdf.

28 Joshi L, Yaganti S, Gemenetzi M, Lightman S, Lindfield D, Liolios $\mathrm{V}$ et al. Dexamethasone implants in retinal vein occlusion: 12-month clinical effectiveness using repeat injections as-needed. Br J Ophthalmol 2013; 97(8): 1040-1044.

29 Bandello F, Parravano M, Cavallero E, Cascavilla ML, Triolo G, Querques L et al. Prospective evaluation of morphological and functional changes after repeated intravitreal dexamethasone implant (Ozurdex) for retinal vein occlusion. Ophthalmic Res 2015; 53(4): 207-216.

30 Feltgen N. Efficacy and safety of ranibizumab $0.5 \mathrm{mg}$ versus dexamethasone $0.7 \mathrm{mg}$ in central retinal vein occlusion: 6-month results of the COMRADE-C study. Invest Ophthalmol Vis Sci 2015; 56: (abstract 5804).

31 Hattenbach L-O. Efficacy and safety of $0.5 \mathrm{mg}$ ranibizumab compared with $0.7 \mathrm{mg}$ dexamethasone intravitreal implant in patients with branch retinal vein occlusion over 6 months: the COMRADE-B study. Invest Ophthalmol Vis Sci 2015; 55: (abstract 1830).

32 Thom HH, Capkun G, Nixon RM, Ferreira A. Indirect comparisons of ranibizumab and dexamethasone in macular oedema secondary to retinal vein occlusion. BMC Med Res Methodol 2014; 14: 140.

33 Pielen A, Feltgen N, Isserstedt C, Callizo J, Junker B, Schmucker C. Efficacy and safety of intravitreal therapy in macular edema due to branch and central retinal vein occlusion: a systematic review. PlOS One 2013; 8(10): e78538.

34 Regnier S, Larsen M, Bezlyak V, Allen F. Comparative efficacy and safety of approved treatments for macular oedema secondary to branch retinal vein occlusion: a network meta-analysis. BMJ Open 2015; 5: e007527.

35 Falavarjani KG, Nguyen QD. Adverse events and complications associated with intravitreal injection of anti-VEGF agents: a review of literature. Eye 2013; 27(7): 787-794.

36 Lotery AJ, Regnier S. Patterns of ranibizumab and aflibercept treatment of central retinal vein occlusion in routine clinical practice in the USA. Eye 2015; 29(3): 380-387.
37 Vandenbroucke JP, von Elm E, Altman DG, Gotzsche PC, Mulrow CD, Pocock SJ et al. Strengthening the reporting of observational studies in epidemiology (STROBE): explanation and elaboration. PLOS Med 2007; 4(10): e297.

38 Kupka K. International Classification of Diseases: Ninth Revision. WHO Chron 1978; 32(6): 219-225.

39 European Medicines Agency. Lucentis (ranibizumab). Summary of product characteristics. Accessed on November 2015. http:/ /www.ema.europa.eu/docs/en_GB/ document_library/EPAR_-_Product_Information/human/ 000715/WC500043546.pdf.

40 Wolf A, Kampik A. Efficacy of treatment with ranibizumab in patients with wet age-related macular degeneration in routine clinical care: data from the COMPASS health services research. Graefe's Arch Clin Exp Ophthalmol 2014; 252(4): 647-655.

41 Food and Drug Administration. Highlights of prescribing information. Ozurdex (dexamethasone). Accessed on November 2015. http:/ / www.accessdata.fda.gov/ drugsatfda_docs/label/2014/022315s009lbl.pdf.

42 Boyer DS, Yoon YH, Belfort Jr R, Bandello F, Maturi RK, Augustin AJ et al. Three-year, randomized, sham-controlled trial of dexamethasone intravitreal implant in patients with diabetic macular edema. Ophthalmology 2014; 121(10): 1904-1914.

43 Acosta R, Hoffmeister L, Román R, Comas M, Castilla M, Castells X. Systematic review of population-based studies of the prevalence of cataracts. Arch Soc Esp Oftalmol 2006; 81: 509-516.

(c) (1) (2) $\Theta$ This work is licensed under a Creative Commons Attribution-NonCommercialNoDerivs 4.0 International License. The images or other third party material in this article are included in the article's Creative Commons license, unless indicated otherwise in the credit line; if the material is not included under the Creative Commons license, users will need to obtain permission from the license holder to reproduce the material. To view a copy of this license, visit http://creativecommons.org/licenses/by-nc-nd/4.0/

(C) The Author(s) 2017

Supplementary Information accompanies this paper on Eye website (http://www.nature.com/eye) 